\title{
Divergence Control of High-Harmonic Generation
}

\author{
Sylvianne Roscam Abbing,,${ }^{1,}$ Filippo Campi, ${ }^{1}$ Faegheh S. Sajjadian, ${ }^{1}$ Nan Lin $\odot,{ }^{2}$ Peter Smorenburg, ${ }^{2}$ \\ and Peter M. Kraus $\oplus^{1, \dagger}$ \\ ${ }^{1}$ Advanced Research Center for Nanolithography, Science Park 106, 1098 XG Amsterdam, Netherlands \\ ${ }^{2}$ ASML Research, ASML Netherlands B.V., 5504 DR Veldhoven, Netherlands
}

(Received 23 December 2019; revised manuscript received 22 March 2020; accepted 6 April 2020; published 12 May 2020)

\begin{abstract}
We show that the divergence of extreme ultraviolet pulses from high-harmonic generation, which is directly linked to the shape and size of the refocused beam, can be controlled by the relative delay between the fundamental and its intense orthogonally polarized second harmonic in two-color high-harmonic generation. We find that the divergence is minimized close to the delays where the number of emitted photons is maximized. These findings are rationalized as the suppression and enhancement of long and short electron trajectories as a function of the phase of the two-color laser field, respectively. The orthogonally polarized second harmonic introduces a lateral-momentum component that can select one trajectory, whereas it deflects the other. At the same time, the second harmonic profoundly modifies the tunnelionization process that initiates high-harmonic generation, which provides another trajectory gate. Our scheme for controlling the divergence facilitates imaging and metrology of embedded structures through the associated reduction of the focus size. In addition, the reduction of the focus size is beneficial for extreme ultraviolet nonlinear optics and spectroscopy.
\end{abstract}

DOI: 10.1103/PhysRevApplied.13.054029

\section{INTRODUCTION}

High-harmonic generation (HHG) $[1,2]$ is the cornerstone of attosecond science [3-6] and equally important for table-top coherent diffraction applications [7-10]. Many spectroscopy, imaging, and possibly industrial-metrology applications $[11,12]$ strictly require small focus sizes. In particular, semiconductor wafer metrology requires microfocusing with limited energy in the wings, which would otherwise produce diffraction artifacts from metrology targets that are oftentimes embedded into an integratedcircuit infrastructure [11]. Furthermore, larger divergent beams reduce the numerical aperture of an optical system.

Microfocusing of broadband pulses from HHG can be achieved by ellipsoidal [13] or multiple toroidal grazingincidence mirrors $[14,15]$ and subsequent focus $[16]$ and wave-front sensing [17] for optimizing optics alignment. However, the quantum nature of the HHG process poses limitations to the focusing of the pulses from HHG: when an electron is ionized in a strong laser field, the

\footnotetext{
*roscam@arcnl.nl

†kraus@arcnl.nl

Published by the American Physical Society under the terms of the Creative Commons Attribution 4.0 International license. Further distribution of this work must maintain attribution to the author(s) and the published article's title, journal citation, and DOI.
}

electron accumulates an intensity-dependent phase during propagation in the continuum, which is imprinted on the emitted high harmonics following electron-ion recombination [18-20]. The typical Gaussian intensity distribution of the driving field in focus [Fig. 1(a)] therefore gives rise to a double-Gaussian divergence-resolved profile of HHG (Fig. 1(b), [21-24]), because each emitted harmonic can be ascribed to a set of two discrete quantum paths, the short and long electron trajectories. These trajectories have a unique transit time of the continuum electron and thus a well-defined dipole phase that is imprinted on the emission.

This origin of divergence in HHG has been understood in a number of early seminal publications [20-23,25]. However, it only became clear very recently that the different trajectories give rise to aberrations, which hinder refocusing of the pulses from HHG to high intensities [26,27]. This is caused by the different virtual sources of the long and short trajectories even if they correspond to the same photon energy [illustrated in Fig. 1(a)]. Therefore, the refocusing of pulses from HHG does not give a clean Gaussian profile but, rather, a double-Gaussian focus, where the long and short trajectories effectively have different focal planes [Fig. 1(c)]. This makes a strong demagnification of the focused beam challenging. The situation becomes worse when considering the broadband spectra associated with HHG. Every harmonic order and every trajectory have different virtual sources, which give rise to strong chromatic 


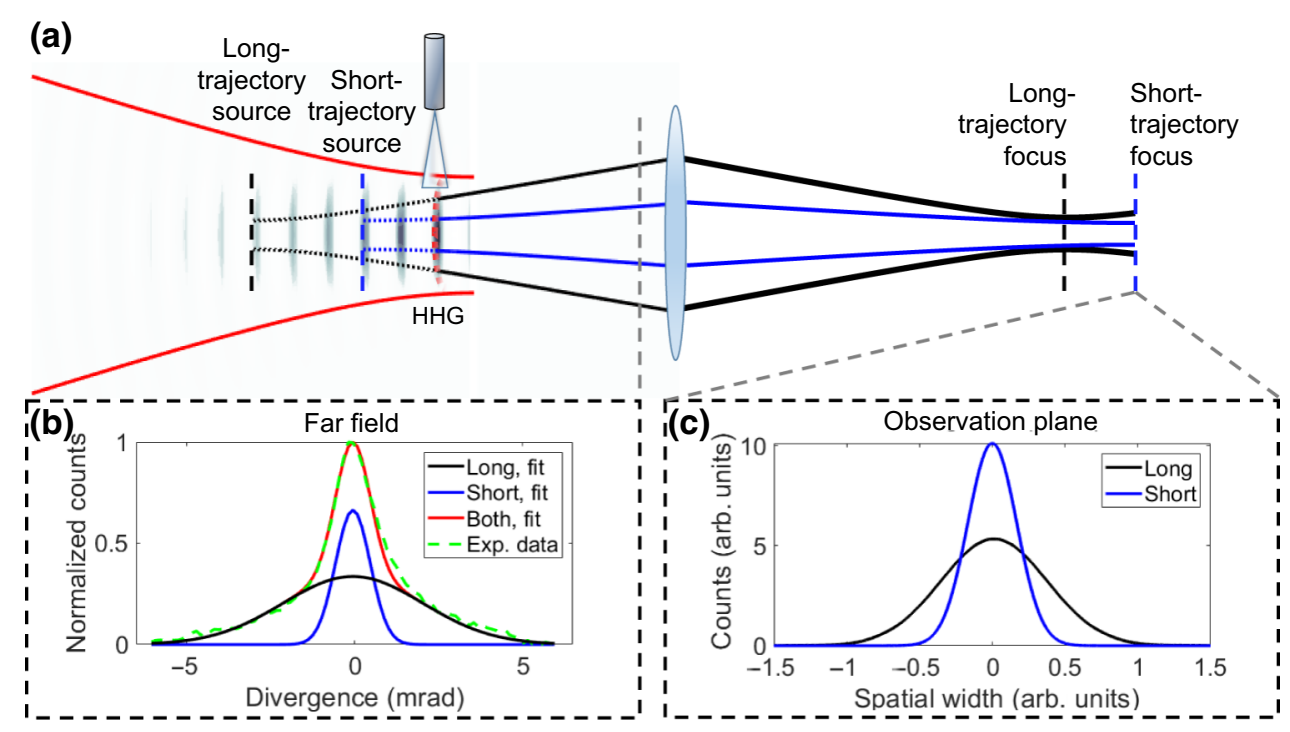

FIG. 1. Virtual sources and foci in HHG. (a) A schematic representation of an HHG source and refocusing of the harmonics (a lens is shown for simplicity). For a given harmonic order $q$, the different contributions of the dipole phases for the short and the long trajectories give rise to different positions of the corresponding virtual sources (dashed blue and black lines). When the harmonics are refocused, the image of the long trajectories will be out of focus, leading to an overall smearing of the profile in the observation plane. (b) An experimental (green dashed line) spectrally integrated divergence-resolved intensity profile in the far field, fitted with a doubleGaussian distribution (red solid line), representing the contributions of short (blue line) and long (black line) trajectories, respectively. (c) The qualitative intensity distribution in the observation (focal) plane, obtained by Fraunhofer diffraction. An additional quadratic radial phase distribution is added to the profile for the long trajectories to account for the larger dipole phase of the long trajectories. This additional phase gives rise to the different divergences and virtual source positions of the long and short trajectories.

aberrations of HHG pulses [26,27]. The double-Gaussian far-field divergence, just like the different virtual sources, are both phenomena that arise due to the existence of both long and short trajectories. Consequently, robust methods to control the divergence are needed, which should simultaneously maintain or improve the overall flux.

In this article, we demonstrate that orthogonally polarized two-color HHG can minimize the divergence and improve the flux at the same time by tuning the relative phase of the two-color field to selectively enhance the short- over the long-trajectory emission.

Our work in this paper builds on a number of earlier papers on two-color HHG. The second harmonic in two-color HHG introduces an additional momentum to the strong-field driven electron in the continuum. This approach has enabled the reconstruction of electron transit times $[28,29]$ using a weak second harmonic for both short and long trajectories [30]. Two-color HHG with a strong second harmonic [31] has enabled the selection of long or short electron trajectories by tuning the relative phase of the two-color pulses. [32]. However, it remained unclear if and how much this strategy would change the divergence, possibly at the expense of the overall conversion efficiency (CE). In a separate work, two-color HHG has been shown to improve the flux of plateau harmonics dramatically [33]. However, in Ref. [33], the cutoff is not detected by the spectrometer, which makes any quantitative assessment of the total flux difficult. Parallel polarized multicolor fields beyond the fundamental and the second harmonic have been numerically demonstrated to simultaneously increase the yield [34] and decrease the divergence of HHG $[35,36]$. Similar multicolor HHG schemes have been experimentally demonstrated to increase the HHG yield [37], as originally proposed in Ref. [38].

\section{EXPERIMENTAL CONCEPT AND SETUP}

In our experiments, we use an 800-nm titanium:sapphire (Ti:Sa) laser with a $40-$ fs pulse duration and a $1-\mathrm{kHz}$ repetition rate. The orthogonally polarized two-color field is generated with a $0.2-\mathrm{mm}$-thick $\beta$-bariumborate (BBO) crystal that produces up to $25 \%$ relative intensity of the second harmonic. We choose the second harmonic (400 $\mathrm{nm}$ ) for controlling the divergence, as it allows for easy and robust implementation. After the BBO, two calcite plates compensate for the group delay between the 800and $400-\mathrm{nm}$ pulses and a pair of fused silica wedges is used for fine adjustment of the group and phase delay of the two-color field. Both calcite plates are tilted slightly off normal with opposite angles to compensate for possible spatial walk-offs between the fundamental and the second harmonic due to refraction, which would lead to slightly nonlinear geometries and therefore smear out the beam profiles. One calcite plate is motorized to scan the 
relative two-color phase delay. We have to scan for less than $0.3^{\circ}$ to achieve a $2 \pi$ rad two-color phase shift.

The two-color pulses are focused with $f=50 \mathrm{~cm}$ focal length to intensities of $(1.8 \pm 0.3) \times 10^{14} \mathrm{~W} / \mathrm{cm}^{2}$ and intersect a very thin effusive gas jet of Ar. This configuration minimizes phase mismatch and reabsorption, leading to the observation of a near-single-atom response. The nozzle is connected to an $x y z$-translation stage for fine positioning of the gas jet relative to the focus. Subsequently, the HHG beam is spectrally dispersed with a concave abberation-corrected flat-field grating onto a double-stack microchannel plate detector backed with a phosphor screen. This geometry serves as a far-field spectrometer, where the spectrum is dispersed in the horizontal plane and the beam freely propagates in the vertical direction. High-harmonic generation with orthogonally polarized two-color fields leads to odd-harmonic emission that is polarized along the polarization of the fundamental driving laser $(800 \mathrm{~nm})$ and even-harmonic emission polarized along the polarization of the second harmonic $(400 \mathrm{~nm})$ [39]. All results are systematically compared to HHG with $800 \mathrm{~nm}$ only, obtained by detuning the $\mathrm{BBO}$ to eliminate all second-harmonic conversion. In addition, we verify that the pulse energies of the $800-\mathrm{nm}$ pulses compared to the $(800+400)-n m$ pulses are identical. For these settings, the cutoffs for HHG with $800 \mathrm{~nm}$ only and $800+400 \mathrm{~nm}$ are the same or $800 \mathrm{~nm}$ only produces one harmonic order more. We find that the exact intensity does not influence the divergence very much, but that it does influence the overall flux of the HHG. Therefore, our estimates for the flux improvement with two-color pulses are deliberately very conservative and larger factors might be achievable.

The concept of the experiment is illustrated in Fig. 2, which shows the classically calculated long and short electron trajectories of harmonic order $13(61 \mathrm{~nm})$ in an orthogonally polarized two-color laser field for two different two-color phases in (a) and (b). The figures also show the relative instantaneous strong-field ionization rate in the two-color field [40,41]. Many groups have previously studied the effect of an low-intensity cross-polarized second harmonic on HHG: only when the field is chosen such that the electron is returning to its origin at the time of return, recombination is efficient. That occurs when the initial lateral velocity is compensated by the lateral displacement introduced by the second harmonic [29]. However, in the present work, the field of the second harmonic is strong, so in addition the second harmonic has a profound influence on the strong-field-ionization step. For 0-rad phase [Fig. 2(a)], the long trajectory recombines to the origin and the short trajectory misses the origin. In addition, the ionization rate at the time of birth of the short trajectory is about 1000 times lower than at the maximum of the pulse. For a phase of $0.5 \pi \mathrm{rad}$, the short trajectory recombines and the long trajectory misses its ion. Moreover, the ionization rate at the time of birth of the short trajectory is only 400
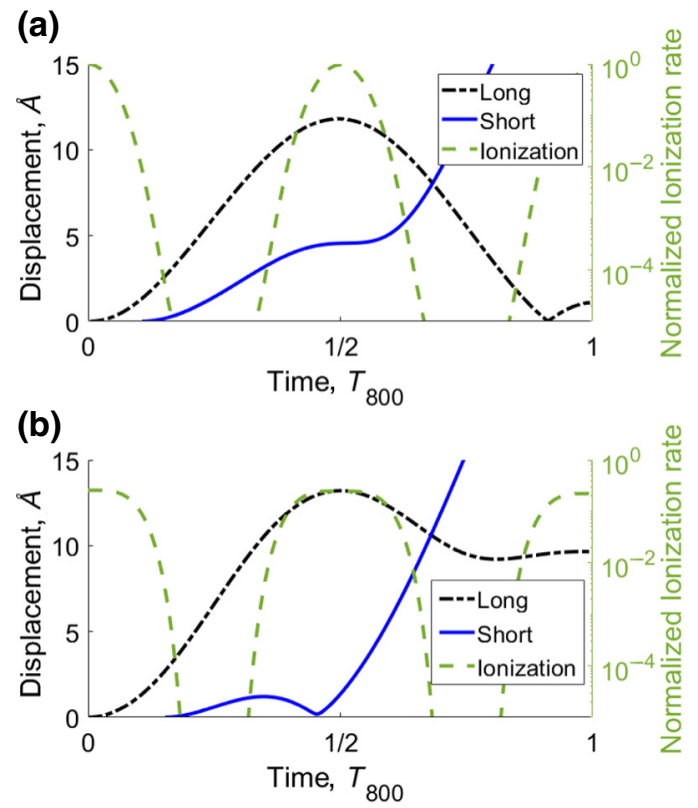

FIG. 2. The concept of trajectory control in two-color HHG. The short (blue) and long (black) trajectories for harmonic 13 (61 $\mathrm{nm})$ as well as the two-color normalized ionization rate (green) are shown for a relative two-color phase of (a) 0 and (b) $0.5 \pi$ $\mathrm{rad}$. For a phase of $0 \mathrm{rad}$ in (a), the short trajectory misses the origin and the long trajectory recombines. The situation is the opposite in (b). In addition, the relative difference in ionization rates (calculated following Ref. [40]) between the long and short trajectories is much less severe in (b) than in (a).

times lower than at the maximum of the pulse. So both ionization and recombination favor the short trajectory for this two-color phase.

While the steering of the continuum trajectories will influence the divergence through trajectory selection, the modulation in the ionization step will have a profound influence on the overall HHG flux. Orthogonally polarized two-color drivers have previously been reported to cause a higher $\mathrm{CE}$ than in conventional one-color HHG [33]. We will show here that these settings of maximum CE correspond to a two-color phase that produces HHG with close to minimum divergence, due to the selection of short trajectories over long trajectories.

\section{RESULTS AND DISCUSSION}

Two-color HHG spectra for two different phases $(0 \mathrm{rad}$ and $0.5 \pi \mathrm{rad}$ ) of the two-color field are shown in Fig. 3 as a function of both the wavelength and the divergence. A relative two-color phase delay of $\pi$ rad corresponds to a delay of about 670 as. In general, two-color HHG leads to the emission of both odd- and even-harmonic orders due to the broken-inversion symmetry of the field. For the HHG conditions in Fig. 3(a), we observe a strongly divergent spectrum, whereas the beam is more collimated for a 

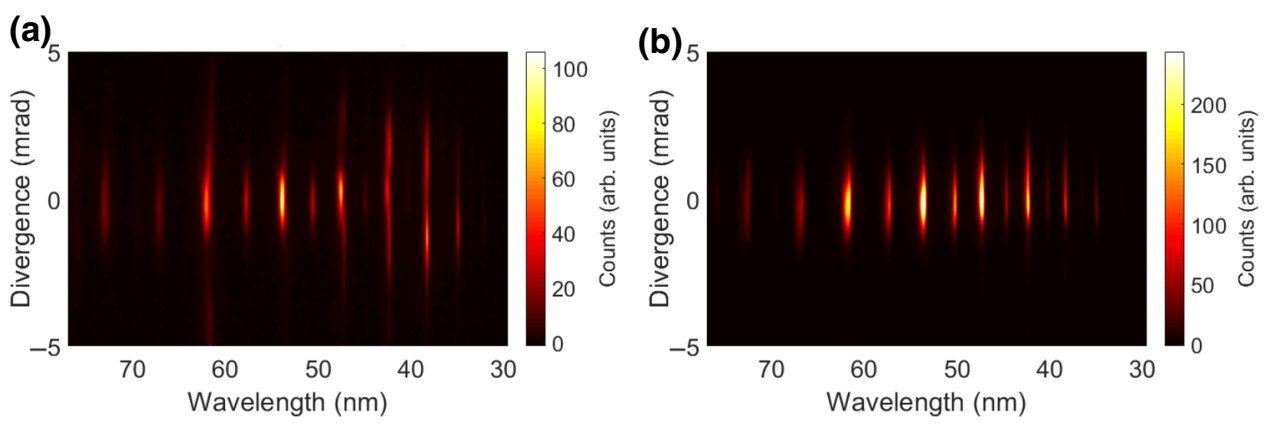

FIG. 3. Far-field HHG spectra for two relative two-color phases. (a) A HHG spectrum for a relative phase of 0 rad, in which long trajectories are selected. (b) A HHG spectrum for a relative phase of $0.5 \pi \mathrm{rad}$, in which short trajectories are selected. The spectrum in (b) shows less signal at higher divergences, higher counts in the central area, and higher overall counts as compared to the spectrum in (a).

two-color phase of $0.5 \pi \mathrm{rad}$, as shown in Fig. 3(b). In addition, the overall number of emitted photons is considerably higher for two-color phases where a more collimated beam is generated [Fig. 3(b)]. As described and simulated in Fig. 2, a two-color phase of 0 rad corresponds to selecting predominantly long trajectories, which explains the more divergent profile in Fig. 3(a), whereas a phase of $0.5 \pi \mathrm{rad}$ selects short trajectories, leading to a less divergent beam profile.

We now turn to the results of a complete scan of the relative phase delay of the two-color field. These results are summarized in Fig. 4. Figure 4(a) is a compact way of plotting an entire scan of a divergence- and energy-resolved HHG spectrum as a function of the two-color phase: the sinusoidal modulations of the HHG signal as a function of the two-color phase [oscillations of a few different energies and divergences are shown in Figs. 4(b)-4(d)] are Fourier transformed pixel by pixel. The frequency component with the highest amplitude from this Fourier transform corresponds to an oscillation-cycle duration of $0.67 \mathrm{fs}$ (equivalent to a relative two-color phase shift by $\pi \mathrm{rad}$ ). The amplitude of this frequency is displayed in Fig. 4(a) as the brightness of the plot. The phase of this frequency is encoded as color in Fig. 4(a). This phase of the Fourier transform is determined by the phase of the intensity oscillations in different pixels [as shown in Figs. 4(b)-4(d)] and can thus be directly linked to relative twocolor delays in units of the phase of the two-color field in radians, or as a delay in femtoseconds, as shown in the legend (round color map) in Fig. 4(a). Two pixels with colors on opposite ends of this legend (e.g., red versus light blue or turquoise) thus correspond to intensity modulations, where the intensity in one pixel is maximized, whereas the other one is minimized for the same twocolor delay. Bright (as opposed to dark) regions indicate an intense harmonic signal.

As explained in Sec. II, the signal of the short trajectories will be enhanced at a different two-color delay than the signal of the long trajectories. The centers of most plateau harmonics (between 50 and $75 \mathrm{~nm}$ ) show a red or purple shading (a relative two-color phase of about 1.2 rad, short trajectories), whereas the regions at larger divergence angles have a light blue color (a phase of about 2.7 $\mathrm{rad}$, long trajectories), which thus indicates that the signals maximize with a time (phase) delay of about $0.32 \mathrm{fs}(1.5$ $\mathrm{rad})$. This shows that the outer and inner parts of the farfield beam in the plateau, which are dominated by long and short trajectories, respectively, modulate completely out of phase (remembering that a relative two-color delay change of $0.67 \mathrm{fs}$, or $\pi \mathrm{rad}$, leads to one full signal oscillation). This color contrast of the outer and inner parts of the farfield profile (e.g., the blue and red colors in the plateau) thus directly visualizes the parts of the far-field beam that are dominated by long and short trajectories, respectively, and is generally a compact way to represent the amplitude and phase of the sinusoidal intensity modulations of two-color delay scans in HHG.

Figures 4(b)-4(d) show the intensity oscillations as a function of the two-color phase in the far field corresponding to selected harmonic orders $12(67 \mathrm{~nm}), 16(50 \mathrm{~nm})$, and $22(36 \mathrm{~nm})$ for both short (squares) and long (circles) trajectories. The phase between two peaks corresponds to $\pi \mathrm{rad}(0.67 \mathrm{fs})$. In Fig. 4(b), the relative delay for maximizing the short or the long trajectory is different by approximately $0.5 \pi \mathrm{rad}(0.33 \mathrm{fs})$, meaning that the short and long trajectories are almost completely out of phase. Comparing harmonic orders 12 in Fig. 4(b) and 16 in Fig. 4(c), we note that this phase difference between the two trajectories becomes smaller for higher harmonics. This effect is even more visible for a harmonic in the cutoff region, as in Fig. 4(d). The long and short trajectories that correspond to higher photon energies are becoming more similar to each other in terms of ionization and recombination times as well as excursion amplitudes and merge in the cutoff, which explains the similar oscillation phases for higher harmonics. Figure 4 thus demonstrates that we can discriminate between the long and short trajectories by adjusting the two-color phase. 
(a)

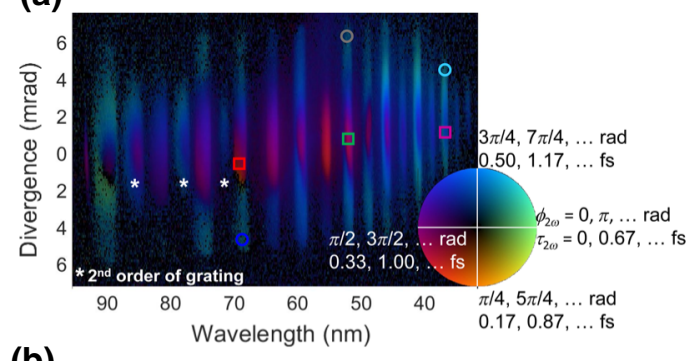

(b)

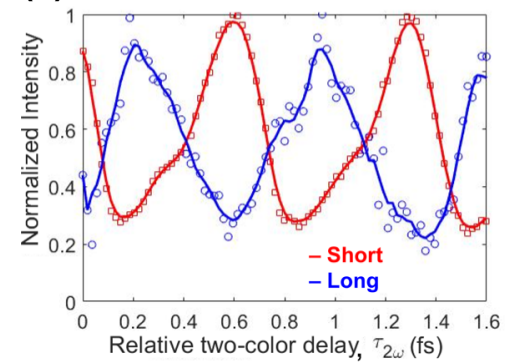

(c)

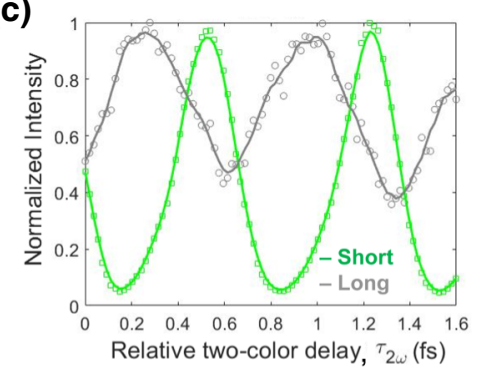

(d)

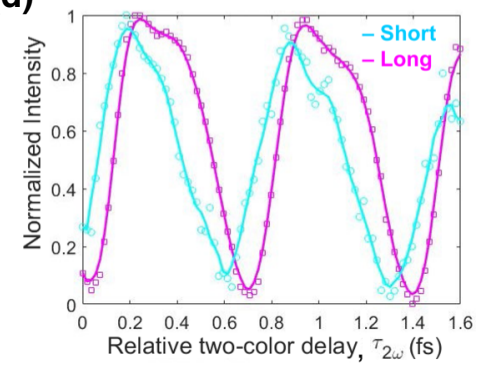

FIG. 4. HHG as a function of the two-color delay. (a) A pixel-by-pixel Fourier transform of the HHG intensity as a function of the two-color delay. The brightness encodes the amplitude of the dominant frequency component of the Fourier transform and the color encodes its phase. The legend (the round color map in the bottom right) indicates how the color is related to the phase (or delay) of the two-color field. The intensity oscillations as a function of the two-color phase for selected regions of interest marked in (a) are shown for (b) harmonic order $12(67 \mathrm{~nm})$, (c) harmonic order $16(50 \mathrm{~nm})$, and (d) harmonic order $22(36 \mathrm{~nm})$ as a function of the two-color delay $\tau_{2 \omega}$ [in fs, a two-color phase shift of $\pi$ rad corresponds to a delay of $0.67 \mathrm{fs}$, as illustrated in the legend of panel (a)]. The open squares correspond to short electron trajectories and the open circles correspond to long trajectories. The open symbols in (b)-(d) are raw data and the lines are five-point moving averages.

We now turn to analyzing and quantifying the divergence change and the signal level of the harmonics for different relative two-color delays in Fig. 5. The divergence of the harmonics is determined by summing the intensity along the wavelength over a region of interest. Figure 5(a) shows beam profiles for a region of interest spanning harmonic 13 through harmonic 23 . The profiles in the main panel are normalized to their maxima to highlight the effect of the two-color fields on the divergence. The intensity of the second harmonic is $25 \%$ of the fundamental. The spectrum is generated with the gas jet before the focus. In this configuration, the divergence of the shorttrajectory contributions is intrinsically small [26]. This small divergence is achieved because the dipole phase and phase-front curvature of the fundamental possess opposite signs and thus partially cancel each other out when the gas jet is placed before the focus. This gas-jet position is therefore the natural choice for divergence optimization in two-color HHG, as the short trajectory component of the beam already possesses the desired property. At the same time, the more divergent long-trajectory contributions are efficiently phase matched with the gas before the focus $[25,42]$. However, we show that this does not present a problem, since the long-trajectory contribution can be effectively suppressed in two-color HHG [32]. The profile of the beam generated with the $800-\mathrm{nm}$ fundamental only (red curve) in Fig. 5(a) is still clearly not monoGaussian, indicating the presence of both long and short trajectories. The blue curve displays the HHG beam profile for a relative two-color phase of $0.5 \pi \mathrm{rad}$, at which the short trajectories are enhanced. The black curve represents the beam profile for a relative phase of $0 \mathrm{rad}$, optimizing the contribution of the long trajectories. The beam generated with an $(800+400)$-nm driver (blue curve) has much less signal in the wings at larger divergence angles in Fig. 5(a) compared to HHG with the fundamental 800-nm field only. Simultaneously, the total signal at this relative phase is enhanced by a factor of 2.2 compared to the HHG with the fundamental field, as can be seen in the inset in Fig. 5(a), which shows the actual and not the normalized intensity. Each beam profile can be fitted by a double Gaussian, as previously illustrated in Fig. 1(b), which allows estimation of the relative contributions from the long and short trajectories. In the case of two-color HHG optimized for short trajectories (blue), the amplitude of the long-trajectory contribution is decreased by a factor of 1.9 compared to the conventional one-color HHG (red). This demonstrates that for a relative phase of $0.5 \pi \mathrm{rad}$, both the divergence and the total signal level are improved. At this phase, the instantaneous ionization rate is modified in favor of the short trajectories and the long trajectories are prevented from recombining. At a relative delay of 0 
(a)

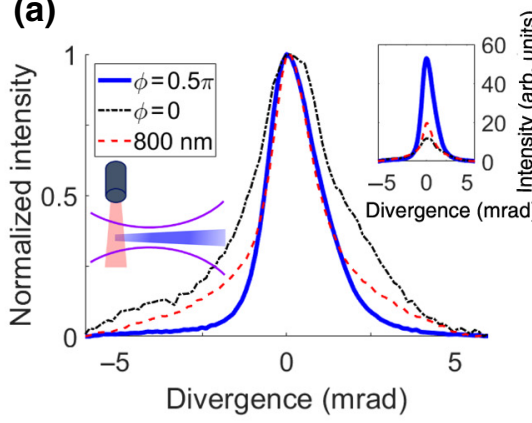

(d)

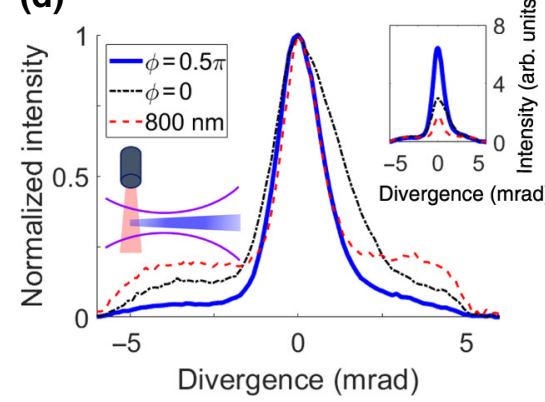

(b)

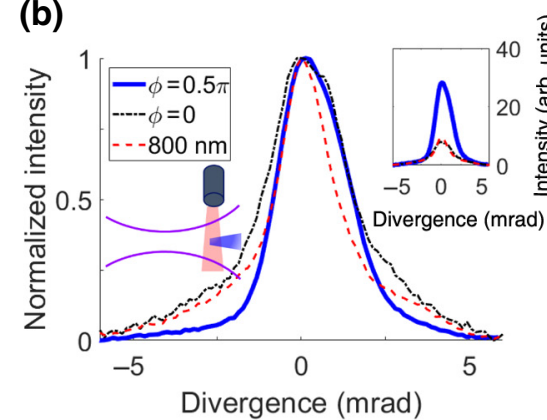

(e)

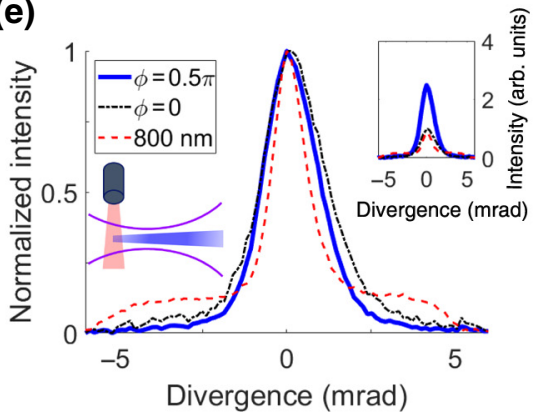

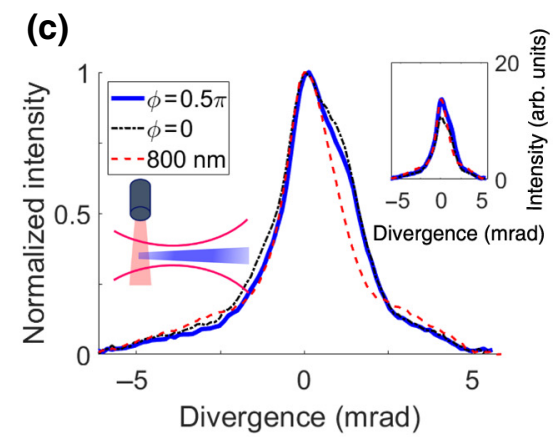

(f)

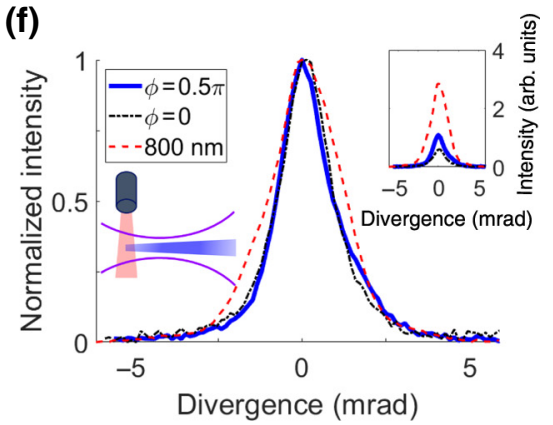

FIG. 5. Divergence control and signal enhancement in two-color HHG. All plots in the main panel show normalized beam profiles for a two-color phase with minimized divergence (solid blue lines, corresponds to predominantly short-trajectory emission), maximized divergence (dash-dotted black lines, corresponds to predominantly long-trajectory emission), and HHG with a one-color 800-nm pulse (dashed red line). The insets show the same profiles with their actual intensities. Panel (a), for the spectrally integrated beam profile from harmonic 13 to 23 , corresponds to the jet being placed before the focus and with $25 \%$ relative intensity of the second harmonic. Panel (b) corresponds to the jet placed after the focus of the two-color pulses under otherwise identical conditions. Panel (c) corresponds to the same geometry as (a), with a weaker second harmonic at $11 \%$ intensity. Panels (d)-(f) correspond to the same measurement as panel (a) and show individual harmonic orders $13(62 \mathrm{~nm}), 14(57 \mathrm{~nm})$, and $23(35 \mathrm{~nm})$.

rad, displayed by the black curve, the signal in the wings is enhanced. In addition, the total signal level is comparable to the signal for the HHG with the fundamental $800-\mathrm{nm}$ field only.

The change of the instantaneous ionization rate as a function of the two-color phase is, in general, small for the long trajectories and, in particular, less significant than for the short trajectories. Therefore, a relative delay of $0 \mathrm{rad}$ does not enhance the signal level of the long trajectories very much. Simultaneously, this phase deflects electrons related to short trajectories, thus decreasing the overall signal from the short trajectories.

Figures $5(\mathrm{~d})-5(\mathrm{f})$ show the beam-profile changes for three separate harmonic orders $13(62 \mathrm{~nm}), 14(57 \mathrm{~nm})$, and $23(35 \mathrm{~nm})$, taken from the same scan as the total profile shown in Fig. 5(a). In order to compare the even harmonics with the fundamental field, an average of the neighboring odd harmonics is taken for generating the red curve in Fig. 5(e). For lower harmonics, the long and short trajectories have vastly different excursion amplitudes. Therefore, the low harmonics show a large difference in total intensity and divergence for the two different relative phases. In all cases, the short-trajectory-optimized $(800+400)$ nm-driven HHG emissions (blue curves) show less signal in the wings, compared to the 800-nm-driven HHG (red curves). Also, the overall intensity (insets) of the blue curve compared to the red curve increases by factors of 10 and 2 for harmonics 13 and 14 [Figs. 5(d) and 5(e)] but decreases for harmonic 23 by about a factor of 2 [Fig. 5(f)]. We note that this harmonic is close to the cutoff and the intensity of the two-color field is likely slightly lower than for $800 \mathrm{~nm}$ only, as observed by a reduced cutoff. However, harmonics near the cutoff show less increase in absolute signal or even a decrease in general. The reason is that the relative excursions for the long and short trajectories become similar for higher harmonics and merge in the cutoff of the spectrum. In addition, the reshaped ionization rate in the two-color field favors a narrower range of trajectories (Fig. 2) and neighboring half-cycles will have very different peak intensities, giving rise to a reduced cutoff of the high-harmonic emission in every second half-cycle. These combined effects cause a reduced yield of cutoff harmonics in two-color HHG.

To further investigate the process of improving the brightness of the HHG beam, the position of the gas jet and the ratio between the power of the fundamental and second-harmonic field are varied. Figure 5(b) shows the beam profiles in which the gas jet is positioned behind 
the focus. In this configuration, phase matching favors the short-trajectory contribution $[25,42]$ but the divergence is increased [26] and the yield decreased [42] compared to the situation in which the jet is before the focus. As a consequence of the intrinsically increased divergence, the signal in the wings is suppressed even more in shorttrajectory-optimized two-color HHG (blue) compared to the fundamental-field case (red), namely by a factor of 2.2 as compared to a factor of 1.9 in Fig. 5(a). The overall intensity of the signal is increased [inset in Fig. 5(b)] by a factor of 2.9, also surpassing the factor of 2.2 obtained in Fig. 5(a), where the gas jet is placed before the focus.

Figure 5(c) shows the beam profiles of an experiment in which the percentage of the second-harmonic power is lowered to $11 \%$. Although the total intensity is slightly improved at a relative delay of $0.5 \pi \mathrm{rad}$ compared to $800 \mathrm{~nm}$ only, the improvement of the divergence is less pronounced with the lower second-harmonic field power. By having a lower contribution of the second harmonic, the ionization rate will be changed less compared to the fundamental-field-only case. Also, the selection of trajectories by introducing a smaller lateral velocity component will become less efficient.

\section{CONCLUSION AND OUTLOOK}

We demonstrate that phase-controlled orthogonally polarized two-color fields can be used to minimize the divergence of HHG, while simultaneously improving the overall flux. The improvement of the divergence is predominantly attributed to the introduction of a lateralmomentum component that enables trajectory selection, while the enhanced number of photons is mainly influenced by the reshaped strong-field ionization rate in a two-color laser field. The suppressed long-trajectory contribution is synonymous with a smaller and cleaner monoGaussian focus when refocusing the harmonics. Such improvements will be hugely beneficial for attosecond science and lenless imaging with HHG sources. Pump-probe experiments with two attosecond pulses from HHG, sometimes dubbed the "holy grail of attosecond science," have made tremendous progress $[43,44]$, but remain challenging because the peak intensities attainable with individual pulses from HHG are relatively low. All experiments that aim at attosecond pump-probe or nonlinear processes in the extreme ultraviolet and soft $\mathrm{x}$-ray range will benefit tremendously from our two-color strategy to achieve brighter HHG with higher-quality foci.

Many imaging applications need a small focus, as too large a field of view can come at the expense of the resolution. In addition, a nonclean (i.e. not mono-Gaussian) focus can cause problems in image-reconstruction algorithms that do not directly retrieve the beam, and it will always cause problems when a fine detail embedded in a larger structure is imaged, where a non-mono-Gaussian focus would create imaging artifacts due to diffraction from the surrounding structures. Improvements of the focusing of HHG are not limited to two-color fields, but extend to any manipulation in the HHG process that favors trajectory selection. This seems to be the beginning of a larger effort, as recent papers have highlighted the importance of understanding and improving $[26,27]$ the microfocusing of HHG [45].

\section{ACKNOWLEDGMENTS}

This work was carried out at the Advanced Research Center for Nanolithography (ARCNL), a public-private partnership of the University of Amsterdam (UvA), the Vrije Universiteit Amsterdam (VU), the Netherlands Organisation for Scientific Research (NWO), and the semiconductor equipment manufacturer ASML. We thank Reinout Jaarsma for technical support. We thank the mechanical workshop and the design, electronic, and software departments of ARCNL for the construction of the setup. P.M.K. acknowledges support from the Netherlands Organisation for Scientific Research (NWO) under Veni Grant No. 016.Veni.192.254.

[1] A. McPherson, G. Gibson, H. Jara, U. Johann, T. S. Luk, I. A. McIntyre, K. Boyer, and C. K. Rhodes, Studies of multiphoton production of vacuum-ultraviolet radiation in the rare gases, JOSA B 4, 595 (1987).

[2] M. Ferray, A. L'Huillier, X. F. Li, L. A. Lompre, G. Mainfray, and C. Manus, Multiple-harmonic conversion of 1064 $\mathrm{nm}$ radiation in rare gases, J. Phys. B 21, L31 (1988).

[3] P. B. Corkum and F. Krausz, Attosecond science, Nat. Phys. 3, 381 (2007).

[4] F. Krausz and M. Ivanov, Attosecond physics, Rev. Mod. Phys. 81, 163 (2009).

[5] P. M. Kraus, M. Zürch, S. K. Cushing, D. M. Neumark, and $\mathrm{S}$. R. Leone, The ultrafast X-ray spectroscopic revolution in chemical dynamics, Nat. Rev. Chem. 2, 82 (2018).

[6] P. M. Kraus and H. J. Wörner, Perspectives of attosecond spectroscopy for the understanding of fundamental electron correlations, Angew. Chem. Int. Ed. 57, 5228 (2018).

[7] R. L. Sandberg, A. Paul, D. A. Raymondson, S. Hädrich, D. M. Gaudiosi, J. Holtsnider, I. T. Raanan, O. Cohen, M. M. Murnane, H. C. Kapteyn et al., Lensless Diffractive Imaging Using Tabletop Coherent High-Harmonic Soft-X-Ray Beams, Phys. Rev. Lett. 99, 098103 (2007).

[8] S. Witte, V. T. Tenner, D. W. Noom, and K. S. Eikema, Lensless diffractive imaging with ultra-broadband table-top sources: From infrared to extreme-ultraviolet wavelengths, Light Sci. Appl. 3, e163 (2014).

[9] M. Zürch, J. Rothhardt, S. Hädrich, S. Demmler, M. Krebs, J. Limpert, A. Tünnermann, A. Guggenmos, U. Kleineberg, and C. Spielmann, Real-time and sub-wavelength ultrafast coherent diffraction imaging in the extreme ultraviolet, Sci. Rep. 4, 7356 (2014). 
[10] D. F. Gardner, M. Tanksalvala, E. R. Shanblatt, X. Zhang, B. R. Galloway, C. L. Porter, R. Karl Jr, C. Bevis, D. E. Adams, H. C. Kapteyn et al., Subwavelength coherent imaging of periodic samples using a $13.5 \mathrm{~nm}$ tabletop high-harmonic light source, Nat. Photonics 11, 259 (2017).

[11] A. J. den Boef, Optical wafer metrology sensors for process-robust $\mathrm{CD}$ and overlay control in semiconductor device manufacturing, Surf. Topogr. 4, 023001 (2016).

[12] H. Kinoshita, T. Harada, Y. Nagata, T. Watanabe, and K. Midorikawa, Development of EUV mask inspection system using high-order harmonic generation with a femtosecond laser, Jpn. J. Appl. Phys. 53, 086701 (2014).

[13] H. Motoyama, T. Sato, A. Iwasaki, Y. Takei, T. Kume, S. Egawa, K. Hiraguri, H. Hashizume, K. Yamanouchi, and H. Mimura, Development of high-order harmonic focusing system based on ellipsoidal mirror, Rev. Sci. Instrum. 87, 051803 (2016).

[14] L. Poletto, F. Frassetto, F. Calegari, S. Anumula, A. Trabattoni, and M. Nisoli, Micro-focusing of attosecond pulses by grazing-incidence toroidal mirrors, Opt. Express 21, 13040 (2013).

[15] H. Coudert-Alteirac, H. Dacasa, F. Campi, E. Kueny, B. Farkas, F. Brunner, S. Maclot, B. Manschwetus, H. Wikmark, J. Lahl, L. Rading, J. Peschel, B. Major, K. Varj, G. Dovillaire, P. Zeitoun, P. Johnsson, A. L'Huillier, and P. Rudawski, Micro-focusing of broadband high-order harmonic radiation by a double toroidal mirror, Appl. Sci. 7, 1159 (2017).

[16] C. Valentin, D. Douillet, S. Kazamias, T. Lefrou, G. Grillon, F. Augé, G. Mullot, P. Balcou, P. Mercère, and P. Zeitoun, Imaging and quality assessment of high-harmonic focal spots, Opt. Lett. 28, 1049 (2003).

[17] L. Freisem, G. Jansen, D. Rudolf, K. Eikema, and S. Witte, Spectrally resolved single-shot wavefront sensing of broadband high-harmonic sources, Opt. Express 26, 6860 (2018).

[18] J. L. Krause, K. J. Schafer, and K. C. Kulander, High-Order Harmonic Generation from Atoms and Ions in the High Intensity Regime, Phys. Rev. Lett. 68, 3535 (1992).

[19] P. B. Corkum, Plasma Perspective on Strong Field Multiphoton Ionization, Phys. Rev. Lett. 71, 1994 (1993).

[20] M. Lewenstein, P. Balcou, M. Y. Ivanov, A. L'Huillier, and P. Corkum, Theory of high-harmonic generation by lowfrequency laser fields, Phys. Rev. A 49, 2117 (1994).

[21] M. Lewenstein, P. Salires, and A. L'Huillier, Phase of the atomic polarization in high-order harmonic generation, Phys. Rev. A 52, 4747 (1995).

[22] M. Bellini, C. Lyngå, A. Tozzi, M. B. Gaarde, T. W. Hänsch, A. L'Huillier, and C.-G. Wahlström, Temporal Coherence of Ultrashort High-Order Harmonic Pulses, Phys. Rev. Lett. 81, 297 (1998).

[23] M. B. Gaarde, F. Salin, E. Constant, P. Balcou, K. J. Schafer, K. C. Kulander, and A. L'Huillier, Spatiotemporal separation of high harmonic radiation into two quantum path components, Phys. Rev. A 59, 1367 (1999).

[24] F. Schapper, M. Holler, T. Auguste, A. Zar, M. Weger, P. Salires, L. Gallmann, and U. Keller, Spatial fingerprint of quantum path interferences in high order harmonic generation, Opt. Express 18, 2987 (2010).
[25] P. Salires, B. Carr, L. L. Droff, F. Grasbon, G. G. Paulus, H. Walther, R. Kopold, W. Becker, D. B. Milosevi, A. Sanpera, and M. Lewenstein, Feynman's path-integral approach for intense-laser-atom interactions, Science 292, 902 (2001).

[26] H. Wikmark, C. Guo, J. Vogelsang, P. W. Smorenburg, H. Coudert-Alteirac, J. Lahl, J. Peschel, P. Rudawski, H. Dacasa, S. Carlström et al., Spatiotemporal coupling of attosecond pulses, Proc. Natl. Acad. Sci. 116, 4779 (2019).

[27] L. Quintard, V. Strelkov, J. Vabek, O. Hort, A. Dubrouil, D. Descamps, F. Burgy, C. Péjot, E. Mével, F. Catoire et al., Optics-less focusing of XUV high-order harmonics, Sci. Adv. 5, eaau7175 (2019).

[28] N. Dudovich, O. Smirnova, J. Levesque, M. Ivanov, D. M. Villeneuve, and P. B. Corkum, Measuring and controlling the birth of attosecond pulses, Nat. Phys. 2, 781 (2006).

[29] D. Shafir, H. Soifer, B. D. Bruner, M. Dagan, Y. Mairesse, S. Patchkovskii, M. Y. Ivanov, O. Smirnova, and N. Dudovich, Resolving the time when an electron exits a tunnelling barrier, Nature 485, 343 (2012).

[30] H. Soifer, M. Dagan, D. Shafir, B. D. Bruner, M. Y. Ivanov, V. Serbinenko, I. Barth, O. Smirnova, and N. Dudovich, Spatio-spectral analysis of ionization times in high-harmonic generation, Chem. Phys. 414, 176 (2013).

[31] X. He, J. Dahlström, R. Rakowski, C. Heyl, A. Persson, J. Mauritsson, and A. L'Huillier, Interference effects in twocolor high-order harmonic generation, Phys. Rev. A 82, 033410 (2010).

[32] L. Brugnera, D. J. Hoffmann, T. Siegel, F. Frank, A. Zaïr, J. W. G. Tisch, and J. P. Marangos, Trajectory Selection in High Harmonic Generation by Controlling the Phase Between Orthogonal Two-Color Fields, Phys. Rev. Lett. 107, 153902 (2011).

[33] I. J. Kim, C. M. Kim, H. T. Kim, G. H. Lee, Y. S. Lee, J. Y. Park, D. J. Cho, and C. H. Nam, Highly Efficient HighHarmonic Generation in an Orthogonally Polarized TwoColor Laser Field, Phys. Rev. Lett. 94, 243901 (2005).

[34] C. Jin, G. Wang, H. Wei, A.-T. Le, and C. Lin, Waveforms for optimal sub-keV high-order harmonics with synthesized two-or three-colour laser fields, Nat. Commun. 5, 1 (2014).

[35] C. Jin, G. J. Stein, K.-H. Hong, and C. D. Lin, Generation of bright, spatially coherent soft $\mathrm{x}$-ray high harmonics in a hollow waveguide using two-color synthesized laser pulses, Phys. Rev. Lett. 115, 043901 (2015).

[36] C. Jin and C. Lin, Control of soft X-ray high harmonic spectrum by using two-color laser pulses, Photonics Res. 6, 434 (2018).

[37] S. Haessler, T. Balčiunas, G. Fan, G. Andriukaitis, A. Pugžlys, A. Baltuška, T. Witting, R. Squibb, A. Zaïr, J. W. G. Tisch, J. P. Marangos, and L. E. Chipperfield, Optimization of Quantum Trajectories Driven by Strong-Field Waveforms, Phys. Rev. X 4, 021028 (2014).

[38] L. E. Chipperfield, J. S. Robinson, J. W. G. Tisch, and J. P. Marangos, Ideal Waveform to Generate the Maximum Possible Electron Recollision Energy for Any Given Oscillation Period, Phys. Rev. Lett. 102, 063003 (2009).

[39] D. Shafir, Y. Mairesse, D. M. Villeneuve, P. B. Corkum, and N. Dudovich, Atomic wavefunctions probed through strong-field light-matter interaction, Nat. Phys. 5, 412 (2009). 
[40] G. L. Yudin and M. Y. Ivanov, Nonadiabatic tunnel ionization: Looking inside a laser cycle, Phys. Rev. A 64, 013409 (2001).

[41] L. V. Keldysh, Ionization in the field of a strong electromagnetic wave, Sov. Phys. JETP 20, 1307 (1965).

[42] P. Salières, A. L'Huillier, P. Antoine, and M. Lewenstein, Study of the spatial and temporal coherence of high-order harmonics, Adv. Atom. Mol. Opt. Phys. 41, 83 (1999).

[43] P. Tzallas, E. Skantzakis, L. A. A. Nikolopoulos, G. D. Tsakiris, and D. Charalambidis, Extreme-ultraviolet pump- probe studies of one-femtosecond-scale electron dynamics, Nat. Phys. 7, 781 (2011).

[44] T. Okino, Y. Furukawa, Y. Nabekawa, S. Miyabe, A. A. Eilanlou, E. J. Takahashi, K. Yamanouchi, and K. Midorikawa, Direct observation of an attosecond electron wave packet in a nitrogen molecule, Sci. Adv. 1, e1500356 (2015).

[45] L. Drescher, O. Kornilov, T. Witting, G. Reitsma, N. Monserud, A. Rouzée, J. Mikosch, M. J. Vrakking, and B. Schütte, Extreme-ultraviolet refractive optics, Nature 564, 91 (2018). 